\title{
Real-World Data for Lenvatinib in Hepatocellular Carcinoma (ELEVATOR): A Retrospective Multicenter Study
}

\author{
Sabrina Welland ${ }^{\mathrm{a}}$ Catherine Leyh $^{\mathrm{b}}$ Fabian Finkelmeier ${ }^{\mathrm{c}} \quad$ André Jefremow $^{\mathrm{d}} \mathrm{e}$ \\ Kateryna Shmanko ${ }^{f}$ Maria A. Gonzalez-Carmona ${ }^{g}$ Arne Kandulski ${ }^{\text {h }}$ Petia Jeliazkova ${ }^{\mathrm{i}}$ \\ Jan Best ${ }^{j}$ Thorben W. Fründt ${ }^{k}$ Angela Djanani' Maria Pangerl ${ }^{\mathrm{m}}$ Andreas Maieron ${ }^{\mathrm{n}}$ \\ Richard Greil $^{\circ}$ Christina Fricke ${ }^{n}$ Disorn Sookthai ${ }^{p}$ Rainer Günther ${ }^{m}$ Andreas Schmiderer ${ }^{l}$ \\ Henning Wege $^{k}$ Marino Venerito $^{q}$ Ursula Ehmer $^{i}$ Martina Müller $^{h}$ \\ Christian P. Strassburg ${ }^{g} \quad$ Arndt Weinmann $^{f} \quad$ Jürgen Siebler ${ }^{\mathrm{e}, \mathrm{r}}$ Oliver Waidmann ${ }^{\mathrm{c}}$ \\ Christian M. Lange ${ }^{\mathrm{b}}$ Anna Saborowski ${ }^{\mathrm{a}}$ Arndt Vogel $^{\mathrm{a}}$ \\ aDepartment of Gastroenterology, Hepatology and Endocrinology, Hannover Medical School, Hannover,

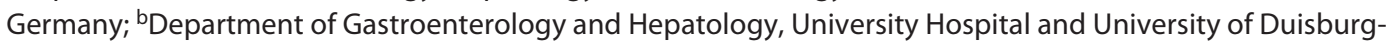 \\ Essen, Essen, Germany; ' Department of Internal Medicine 1, Goethe-University Hospital Frankfurt, Frankfurt, \\ Germany; ${ }^{d}$ Department of Internal Medicine 1, Friedrich-Alexander-Universität Erlangen-Nürnberg (FAU), \\ Erlangen, Germany; ${ }^{\circ} \mathrm{CCC}$-Erlangen EMN, Erlangen, Germany; fDepartment of Medicine I, University Medical Center \\ Johannes Gutenberg University, Mainz, Germany; ${ }^{9}$ Department of Internal Medicine 1, University Hospital of Bonn, \\ Bonn, Germany; hepartment of Internal Medicine I, Regensburg University Hospital, Regensburg, Germany; \\ IInternal Medicine II, Klinikum rechts der Isar, Technical University of Munich, Munich, Germany; 'Department of \\ Internal Medicine, University Hospital Knappschaftskrankenhaus, Ruhr-University Bochum, Bochum, Germany; \\ kDepartment of Gastroenterology and Hepatology, University Medical Center, Hamburg, Germany; 'Department \\ of Gastroenterology, Hepatology and Endocrinology, University Hospital of Innsbruck, Innsbruck, Austria; \\ mDepartment of Internal Medicine I, University Medical Center Schleswig-Holstein (UKSH), Campus Kiel, Kiel,

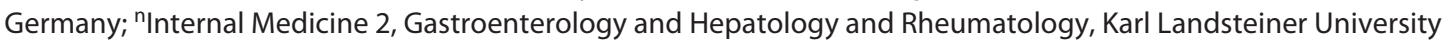 \\ of Health Sciences, University Hospital of St. Pölten, St. Pölten, Austria; ${ }^{\circ}$ Salzburg Cancer Research Institute, \\ Department of Internal Medicine III with Hematology, Medical Oncology, Hemostaseology, Infectiology and \\ Rheumatology, Oncologic Center, Erlangen, Germany; PInstitut für Klinische Krebsforschung IKF GmbH, Frankfurt, \\ Germany; ${ }^{9}$ Department of Gastroenterology, Hepatology and Infectious Diseases, Otto-von-Guericke-University \\ Hospital Magdeburg, Magdeburg, Germany; 'Deutsches Zentrum Immuntherapie (DZI), Erlangen, Germany
}

\section{Keywords}

Lenvatinib · Hepatocellular carcinoma $\cdot$ REFLECT study · Real-world cohort · Liver function · Albumin-bilirubin grade

\begin{abstract}
Background: Lenvatinib is approved as the first-line treatment for patients with advanced hepatocellular carcinoma (HCC). The efficacy of lenvatinib in Caucasian real-world pa-
\end{abstract}

tients is insufficiently defined. The purpose of this study was to evaluate the efficacy of lenvatinib in a multicenter cohort (ELEVATOR) from Germany and Austria. Methods: We conducted a retrospective data analysis of 205 patients treated with first-line systemic lenvatinib at 14 different sites. Overall survival, progression-free survival, overall response rate, and adverse event rates were assessed and analyzed. Results: Pa-

Anna Saborowski and Arndt Vogel contributed equally.
(C) 2022 The Author(s).

Published by S. Karger AG, Basel

This article is licensed under the Creative Commons Attribution 4.0 International License (CC BY) (http://www.karger.com/Services/ OpenAccessLicense). Usage, derivative works and distribution are permitted provided that proper credit is given to the author and the original publisher.
Correspondence to:

Arndt Vogel, vogel.arndt@mh-hannover.de 
tients receiving lenvatinib in the real-world setting reached a median overall survival (mOS) of 12.8 months, which was comparable to the results reported from the REFLECT study. mOS and median progression-free survival (mPFS) were superior in those patients who met the inclusion criteria of the REFLECT study compared to patients who failed to meet the inclusion criteria (mOS 15.6 vs. 10.2 months, hazard ratio [HR] $0.55,95 \%$ confidence interval [95\% Cl]: $0.38-0.81, p=0.002$; mPFS 8.1 vs. 4.8 months, HR $0.65,95 \%$ Cl: $0.46-0.91, p=$ $0.0015)$. For patients with an impaired liver function according to the albumin-bilirubin (ALBI) grade or reduced ECOG performance status $\geq 2$, survival was significantly shorter compared to patients with sustained liver function (ALBI grade 1) and good performance status (ECOG performance status 0 ), respectively (HR 1.69, 95\% Cl: 1.07-2.66, $p=0.023$; HR 2.25, $95 \%$ Cl: $1.19-4.23, p=0.012$ ). Additionally, macrovascular invasion (HR 1.55, 95\% Cl: 1.02-2.37, $p=0.041$ ) and an AFP $\geq 200$ $\mathrm{ng} / \mathrm{mL}$ (HR 1.56, 95\% Cl: 1.03-2.34, $p=0.034$ ) were confirmed as independent negative prognostic factors in our cohort of patients with advanced HCC. Conclusion: Overall, our data confirm the efficacy of lenvatinib and did not reveal new or unexpected side effects in a large retrospective Caucasian real-world cohort, supporting the use of lenvatinib as a meaningful alternative for patients that cannot be treated with IObased combinations in first-line HCC.

(c) 2022 The Author(s).

Published by S. Karger AG, Basel

\section{Introduction}

Hepatocellular carcinoma (HCC) is the third [1] most common cause of cancer death worldwide $[2,3]$, and its incidence is expected to rise. HCC often develops in the fibrotic or cirrhotic liver, and the frequently impaired liver function not only contributes to high mortality but also severely limits treatment options across all stages of the disease.

For many years, the tyrosine kinase inhibitor sorafenib was the only effective systemic therapy available, but in recent years, positive phase-III studies have been reported for the multityrosine kinase inhibitors lenvatinib, cabozantinib, regorafenib, and the vascular endothelial growth factor (VEGF) receptor 2 antibody ramucirumab [4-7]. Lenvatinib is an orally available multityrosine kinase inhibitor with multiple targets, including VEGFR 1-3, fibroblast growth factor receptor 1-4, platelet-derived growth factor receptor $\alpha$, RET, and KIT. Based on the results of the randomized, prospective phase-III REFLECT trial, lenvatinib was approved for the first-line treatment of advanced or unresectable HCC in Europe in August 2018. The RE-
FLECT trial demonstrated noninferiority of lenvatinib compared to sorafenib with a median overall survival (mOS) of 13.6 months for lenvatinib versus 12.3 months for sorafenib (HR 0.92, 95\% CI: 0.79-1.06) [8]. In 2020, the combination of the programmed death ligand 1 antibody atezolizumab and bevacizumab, an antibody that targets VEGF A, was approved for the first-line treatment of unresectable HCC based on the safety and efficacy findings from the phase-III IMbrave150 trial [9]. Two network metaanalyses delineated the superior efficacy of the immuneoncology (IO)-based combination over current tyrosine kinase inhibitors and mono-immunotherapies, supporting the recently updated recommendations of national and international guidelines that consider atezolizumab plus bevacizumab the standard of care for first-line therapy in unresectable HCC patients [10-12]. For lenvatinib, promising activity in several solid tumors including HCC has been demonstrated in combination with pembrolizumab, a concept that is now further evaluated in ongoing trials in intermediate-stage and advanced HCC [13].

Even as the treatment landscape for advanced HCC further evolves with IO-based combinations, TKIs such as lenvatinib will not only remain a relevant alternative for those patients that harbor contraindications to immunotherapies but are also an integral component of sequential treatment strategies. Currently, however, only sorafenib is approved independent of the line of therapy and is therefore the only drug that is reimbursed after lenvatinib or atezolizumab and bevacizumab by all health insurance companies. Reimbursement of regorafenib, cabozantinib, and ramucirumab officially warrants prior therapy with sorafenib, although several health insurance companies in Germany and Austria have adopted the recommendations of current clinical practice guidelines and reimburse all approved HCC drugs also in second line.

In clinical practice, systemic therapies are frequently administered to patients, who do not exactly match the study population of the pivotal trials. The purpose of this study was to evaluate the efficacy and tolerability of lenvatinib as the first-line therapy in a real-world cohort of 205 patients (ELEVATOR cohort) with advanced HCC, many of whom did not meet the inclusion criteria of the REFLECT trial.

\section{Methods}

Population and Data Collection

Data from 205 patients with with histologic or image-based diagnosis of HCC that were started on first-line systemic therapy with lenvatinib between October 2017 and September 2020 were 
Table 1. Baseline characteristics

\begin{tabular}{|c|c|c|c|}
\hline & $\begin{array}{l}\text { Overall } \\
(n=205)\end{array}$ & $\begin{array}{l}\text { REFLECT-in } \\
(n=110)\end{array}$ & $\begin{array}{l}\text { REFLECT-out } \\
(n=95)\end{array}$ \\
\hline \multicolumn{4}{|l|}{ Age, years } \\
\hline Mean & 67.7 (SD 10.5) & 68.9 (SD 10.6) & 66.3 (SD 10.2) \\
\hline Median & 69 & 71 & 67 \\
\hline \multicolumn{4}{|l|}{ Sex } \\
\hline Male & $167(81.5)$ & $87(79.1)$ & $80(84.2)$ \\
\hline Female & $38(18.5)$ & $23(20.9)$ & $15(15.8)$ \\
\hline \multicolumn{4}{|l|}{ Weight } \\
\hline$<60 \mathrm{~kg}$ & $20(9.7)$ & $14(12.7)$ & $6(6.4)$ \\
\hline$>60 \mathrm{~kg}$ & $177(86.3)$ & $91(82.7)$ & $86(90.5)$ \\
\hline No data available & $8(4)$ & $5(4.6)$ & $3(3.1)$ \\
\hline \multicolumn{4}{|l|}{ Vital status at last follow-up } \\
\hline Dead & $110(53.7)$ & $53(48.2)$ & $57(60.0)$ \\
\hline Alive & $76(37.1)$ & $46(41.8)$ & $30(31.6)$ \\
\hline Lost to follow-up & $19(9.2)$ & $11(10.0)$ & $8(8.4)$ \\
\hline \multicolumn{4}{|l|}{ Follow-up time, months } \\
\hline Mean & 10.7 & 12.2 & 8.4 \\
\hline Median & 8.8 & 9.9 & 6.9 \\
\hline \multicolumn{4}{|c|}{ ECOG performance status at baseline } \\
\hline 0 & $85(41.5)$ & $52(47.3)$ & $33(34.7)$ \\
\hline 1 & $91(44.4)$ & $55(50.0)$ & $36(37.9)$ \\
\hline 2 & $23(11.2)$ & 0 & $23(24.3)$ \\
\hline$>2$ & 0 & 0 & 0 \\
\hline No data available & $6(2.9)$ & $3(2.7)$ & $3(3.1)$ \\
\hline \multicolumn{4}{|l|}{ BCLC status at baseline } \\
\hline BCLC A (early) & $6(2.9)$ & $4(3.6)$ & $2(2.1)$ \\
\hline BCLC B (intermediate) & $58(28.3)$ & $41(37.3)$ & $17(17.9)$ \\
\hline BCLC C (advanced) & $141(68.8)$ & $65(59.1)$ & $76(80.0)$ \\
\hline \multicolumn{4}{|l|}{ Child-Pugh score at baseline } \\
\hline Child-Pugh A & $160(78.4)$ & $110(100)$ & $50(52.6)$ \\
\hline Child-Pugh B & $45(21.6)$ & 0 & $45(47.4)$ \\
\hline Child-Pugh C & 0 & 0 & 0 \\
\hline \multicolumn{4}{|l|}{ ALBI grade at baseline } \\
\hline ALBI 1 & $70(34.1)$ & $51(46.4)$ & $19(20.0)$ \\
\hline ALBI 2 & $116(56.6)$ & $55(50.0)$ & $61(64.2)$ \\
\hline ALBI 3 & $19(9.3)$ & $4(3.6)$ & $15(15.8)$ \\
\hline \multicolumn{4}{|l|}{ Etiology } \\
\hline Alcohol related & $52(25.3)$ & $26(23.6)$ & $26(27.4)$ \\
\hline Hepatitis B & $26(12.7)$ & $12(10.9)$ & $14(14.7)$ \\
\hline Hepatitis C & $32(15.6)$ & $18(16.4)$ & $14(14.7)$ \\
\hline Nonalcoholic steatohepatitis & $42(20.5)$ & $23(20.9)$ & $19(20.0)$ \\
\hline Others & $12(5.9)$ & $4(3.6)$ & $8(8.5)$ \\
\hline Unknown & $41(20.0)$ & $27(24.6)$ & $14(14.7)$ \\
\hline \multicolumn{4}{|c|}{ Presence of portal vein thrombosis/extrahepatic spread } \\
\hline Main portal vein invasion & $47(22.9)$ & 0 & $47(49.5)$ \\
\hline Extrahepatic spread & $80(39.0)$ & $46(41.8)$ & $34(35.8)$ \\
\hline PVI, EHS, or both & $112(54.6)$ & & \\
\hline \multicolumn{4}{|l|}{ AFP at baseline } \\
\hline AFP $\geq 200 \mathrm{ng} / \mathrm{mL}$ & $87(42.4)$ & $38(34.5)$ & $49(51.6)$ \\
\hline AFP $\geq 400 \mathrm{ng} / \mathrm{mL}$ & $75(36.6)$ & $31(28.2)$ & $44(46.3)$ \\
\hline Median AFP, ng/mL & 58.8 & 16.6 & 349 \\
\hline
\end{tabular}

Real-World Data for Lenvatinib in Hepatocellular Carcinoma
Liver Cancer 2022;11:219-232

DOI: $10.1159 / 000521746$ 
Table 1 (continued)

\begin{tabular}{llll}
\hline & $\begin{array}{l}\text { Overall } \\
(n=205)\end{array}$ & $\begin{array}{l}\text { REFLECT-in } \\
(n=110)\end{array}$ & $\begin{array}{l}\text { REFLECT-out } \\
(n=95)\end{array}$ \\
\hline $\begin{array}{l}\text { Previous treatment } \\
\text { Any previous anticancer procedure }\end{array} \quad$ Rection & $148(72.2)$ & $88(80.0)$ & $60(63.2)$ \\
$\quad$ Transplantation & $53(25.8)$ & $37(33.6)$ & $16(16.8)$ \\
Radiation & $4(1.9)$ & $4(3.6)$ & 0 \\
Ablation & $6(2.9)$ & $3(2.7)$ & $3(3.1)$ \\
SIRT & $46(22.4)$ & $29(26.4)$ & $17(17.9)$ \\
TACE & $31(15)$ & $21(19.1)$ & $10(10.5)$ \\
No previous treatment & $87(42.4)$ & $46(41.8)$ & $41(43.2)$ \\
Lenvatinib dose at baseline & $57(27.8)$ & $22(20)$ & $35(36.8)$ \\
Standard dose at baseline & & $52(47.3)$ & $37(38.9)$ \\
Reduced dose at baseline & $89(43.4)$ & $56(50.9)$ & $58(61.1)$ \\
No data available & $114(55.6)$ & $2(1.8)$ & 0 \\
\hline
\end{tabular}

Baseline characteristics of patients that meet (REFLECT-in) or do not meet the inclusion criteria of the REFLECT trial (REFLECT-out).

collected retrospectively from 14 centers in Germany and Austria. In agreement with a real-world dataset, no particular inclusion and exclusion criteria were defined except for absence of prior systemic HCC therapy. Specifically, in contrast to the REFLECT trial, patients with a Child-Pugh (CP) score $>$ A and Eastern Cooperative Oncology Group (ECOG) performance status $>1$ by definition of Oken et al. [14], bile duct invasion, main portal vein invasion, and a tumor burden $>50 \%$ were not excluded from the analysis.

Baseline characteristics, including age, gender, weight, underlying liver disease, and tumor-specific characteristics such as date of diagnosis, tumor stage at diagnosis according to the Barcelona clinical liver cancer (BCLC) classification, macrovascular and portal vein invasion, extrahepatic spread, and previous treatments, were assessed retrospectively. The starting dose of lenvatinib and dose interruptions during the course of treatment were documented. Adverse events (AEs) were graded according to the common terminology criteria for adverse events version 5.0. Liver function was classified according to the $\mathrm{CP}$ score and graded according to the albumin-bilirubin (ALBI) score at start and end of treatment. Treatment response was evaluated by the best radiological response under treatment by computed tomography or magnetic resonance imaging. Response was categorized as complete and partial response (CR and $\mathrm{PR}$ ), stable disease (SD), and progressive disease (PD) by local review according to Response Evaluation Criteria in Solid Tumors (RECIST) 1.1. Disease control rate (DCR) was defined as the proportion of patients achieving a CR/PR or SD as the best radiological response. In addition to radiological response, alpha-fetoprotein (AFP) values at baseline and the AFP nadir during the course of treatment were documented. Apart from tumor progression, other reasons for treatment discontinuation such as liver function deterioration and other AEs were analyzed.

Patients were followed until death or data cutoff (February 28, 2021). Patients that were alive but with the last documented visit more than 6 months before data cutoff were considered lost to follow-up.

\section{Statistical Analysis}

Statistical analysis was performed with IBM SPSS statistics version 25 (SPSS Inc., Chicago, IL, USA). Baseline characteristics were described as numbers, percentages, and median with ranges. Overall survival (OS) was defined by the date of treatment start until the day of death or the follow-up deadline mentioned above, respectively. The time between lenvatinib start and date of first radiologic proven PD was used for progression-free survival (PFS). Median OS and PFS was calculated by the Kaplan-Meier method. $\chi^{2}$ and log-rank tests were used to analyze differences in OS and PFS. Relative risks were expressed as hazard ratio (HR) with a $95 \%$ confidence interval (95\% CI). For identification of independent risk factors, a multivariate analysis using the Cox proportional hazard model with stepwise backwards elimination was used. For all analyses, a significance level of $p<0.05$ was considered significant. The degree of correlation between categorical and not normal-distributed steady variants was calculated by the Spearman-Rho test for categorical and steady variables while the Phi coefficient was used for the correlation test of nominal variables.

\section{Results}

\section{Patient Characteristics}

A total of 205 patients, 198 Caucasian and 7 non-Caucasian, from 14 sites who received lenvatinib as systemic first-line treatment were included in the analysis of the ELEVATOR real-world cohort. During the median follow-up time of 8.8 months (mean 10.4 months, $0.63-36.4$ months), 110 patients died (53.7\%). Seventy-six patients were alive at the time of the last survey, of whom 24 continued to receive lenvatinib. Nineteen cases were lost to follow-up. The median age at treatment initiation was 69 
Table 2. Treatment characteristics of patients allocated to the REFLECT-in and REFLECT-out subgroups at baseline

\begin{tabular}{llll}
\hline & Overall $(n=205)$ & REFLECT-in $(n=110)$ & REFLECT-out $(n=95)$ \\
\hline Best response under lenvatinib treatment & & & \\
$\quad$ Complete response & $1(0.4)$ & 0 & $1(1.1)$ \\
$\quad$ Partial response & $47(22.9)$ & $28(25.5)$ & $19(20.0)$ \\
$\quad$ Stable disease & $69(33.7)$ & $44(40.0)$ & $25(26.3)$ \\
$\quad$ Progressive disease & $43(20.9)$ & $18(16.4)$ & $25(26.3)$ \\
End of treatment before first imaging & $38(18.6)$ & $16(14.5)$ & $22(23.2)$ \\
No data available & $7(3.5)$ & $4(3.6)$ & $3(3.1)$ \\
Median overall survival, months & 12.8 & 15.6 & 10.2 \\
Median progression-free survival, months & 6.4 & 8.1 & 4.8 \\
Disease control & $117(57.1)$ & $71(64.5)$ & $46(48.4)$ \\
Overall response & $48(23.4)$ & $28(25.5)$ & $20(21.1)$ \\
AFP reduction $>$ 20\% under treatment & $57(27.8)$ & $24(21.8)$ & $33(34.7)$ \\
Reason for end of treatment & & & \\
Progression & $93(45.5)$ & $51(46.4)$ & $42(44.1)$ \\
Adverse events & $75(36.5)$ & $36(32.7)$ & $39(41.0)$ \\
$\quad$ Liver function deterioration & $30(14.6)$ & $10(9.1)$ & $20(21.0)$ \\
$\quad$ Other adverse events & $45(21.9)$ & $26(23.6)$ & $19(20.0)$ \\
Patient choice & $3(1.4)$ & $2(1.8)$ & $1(1.1)$ \\
Others & $4(2)$ & $2(1.8)$ & $2(2.2)$ \\
No data available & $6(2.9)$ & $4(3.6)$ & $2(2.2)$ \\
Treatment ongoing & $24(11.7)$ & $15(13.6)$ & $9(9.3)$ \\
\hline
\end{tabular}

Lenvatinib treatment characteristics, patients stratified according to REFLECT-in and REFLECT-out.

years, and $81.5 \%$ of the patients were male. The majority of the patients had ECOG performance status of 0 or 1 . Most patients had a preserved hepatic function as assessed by CP score and ALBI grade: $78.4 \%$ of patients were classified as $\mathrm{CP}$ A and the remaining $21.6 \%$ as $\mathrm{CP}$ B. In respect to the ALBI score, ALBI grade 2 (56.6\%) was most prominent, followed by ALBI grade $1(34.1 \%)$ and 3 (9.3\%) (Table 1). In the CP A cohort, $43.75 \%$ fell into the ALBI grade 1 category and $56.25 \%$ were classified as ALBI grade 2.

In $68.8 \%$ of cases, treatment was initiated at advanced stage. Portal vein invasion was present in $22.9 \%$, and extrahepatic spread was evident in $39 \%$ of cases. The median AFP value was $58.80 \mathrm{ng} / \mathrm{mL}$ (mean 13,333.53 ng/ $\mathrm{mL}$ ), while $42.4 \%$ of patients presented with an AFP value $\geq 200 \mathrm{ng} / \mathrm{mL}$ at baseline and in $36.6 \% \geq 400 \mathrm{ng} / \mathrm{mL}$ (Table 1).

According to the inclusion criteria, none of the patients had received prior systemic HCC treatment, but 148 patients had undergone various anticancer procedures. Overall, only $27.8 \%$ of patients were treatment naïve, and systemic therapy was initiated as first tumor treatment (Table 1).

In order to assess the impact of the REFLECT inclusion and exclusion criteria on safety and efficacy of len-

Real-World Data for Lenvatinib in

Hepatocellular Carcinoma vatinib, the cohort was split into the REFLECT-in ( $n=$ $110)$ and the REFLECT-out ( $n=95)$ subgroups. According to the REFLECT inclusion criteria, patients with CP $A$ and an ECOG performance status of 1 or better were considered "REFLECT-in," whereas all patients who did not meet the criteria of the pivotal trial, for instance patients with invasion of the main portal vein, were regarded as REFLECT-out.

Baseline characteristics of patients in the REFLECT-in and -out subgroup were similar with respect to age, gender distribution, and weight. According to the exclusion criteria of the REFLECT trial, liver function at baseline was worse in the REFLECT-out compared to the REFLECT-in subgroup. In addition to the impaired liver function, patients in the REFLECT-out subgroup suffered from more advanced disease at the time of lenvatinib initiation and had higher AFP levels. Of note, a lower proportion of the REFLECT-out group had received prior local treatments compared to the REFLECT-in population (Table 1).

A total of 114 patients (55.6\%) in the ELEVATOR cohort received lenvatinib at a starting dose that was lower than the body weight-adapted recommendation, and only 89 patients $(43.3 \%)$ received the recommended dose at the time of treatment initiation (Table 1). Discontinu- 
ation of lenvatinib treatment was due to progression on therapy in $45.5 \%$, whereas medication was discontinued in $36.5 \%$ of cases due to AEs, with deterioration of liver function in $14.6 \%$ of patients and other AEs causing discontinuation in $21.9 \%$ (Table 2). Average time on treatment was 6.0 months (median 4.1 months).

\section{Efficacy}

mOS was 12.8 months (95\% CI: 10.9-14.7, mean 17.1 months) with a mPFS of 6.4 months (95\% CI: 5.1-7.7, mean 9.5 months). The overall response rate was $23.4 \%$ and the DCR 57.1\% (Table 2). Forty-three patients (20.9\%) had PD as the best response in the first CT-scan. Lenvatinib treatment was discontinued due to AEs before the first staging in 38 cases (18.6\%) with no information on tumor response (Table 2).

In the REFLECT-in subgroup, mOS was 15.6 months (95\% CI: 10.7-20.5, mean 19.4 months) and thereby significantly exceeded the mOS in the REFLECT-out subgroup (mOS 10.2 months, 95\% CI: 7.9-12.5, $p=0.002$, mean 11.9 months) (Table 3; Fig. 1b). The mPFS with lenvatinib in the REFLECT-in cohort was 8.1 months, while the mPFS was 4.8 months in the REFLECT-out subgroup $(p=0.011)$ (Table 2; Fig. 1a). ORR (25.5\% vs. $21.1 \%$, $p=0.729)$ and DCR (64.5\% vs. $48.4 \%, p=0.063)$ also tended to be higher in the REFLECT-in compared to the RELFECT-out patients, respectively. In respect to underlying liver disease, $\mathrm{mOS}$ was 12.8 months in patients with HBV, 13.2 months with HCV, 12.6 months with alcoholic steatohepatitis, and 10.2 months with nonalcoholic steatohepatitis.

Since a relevant number of patients in our cohort received a lower lenvatinib dose at treatment start compared to patients in the REFLECT study, we investigated to which extent the dose reduction affected treatment outcome. The mPFS of patients with upfront dose reduction was not significantly lower compared to standard dose lenvatinib patients (5.9 months [95\% CI: 4.5-7.3 months], mean 9.2 months vs. 6.7 months [95\% CI: 4.88.6], mean 9.1 months, $p=0.79)$. Furthermore, DCR and ORR were also similar (DCR: $59.6 \%$ vs. $53.9 \%, p=0.207$; ORR: $21.9 \%$ vs. $25.8 \%, p=0.258$ ) (online suppl. Table 1 ; for all online suppl. material, see www.karger.com/ doi/10.1159/000521746).

Next, treatment efficacy was evaluated according to baseline liver function. Patients with good liver function (ALBI grade 1) had a significantly longer mOS than patients with ALBI grade 2 or ALBI grade 3 liver function (ALBI grade 1: 19.9 months, ALBI grade 2: 11.2 months, and ALBI grade 3: 7.1 months, $p=0.001$ ) (Table 4; Fig. 2b).
ALBI grade 1 patients also outperformed ALBI grade 3 patients in terms of PFS (8.6 months vs. 4.9 months, $p=$ 0.0015), but the PFS difference did not reach statistical significance when comparing ALBI grade 1 to ALBI grade 2 (8.6 months vs. 5.9 months; $p=0.277$ ) (Table 4; Fig. 2a). A higher response rate was observed in patients with better liver function (ORR ALBI grade 1: $31.4 \%$ vs. ORR ALBI grade 2 and 3: 19.26\%, $p=0.05$ ) (Table 4).

In our cohort, OS was independent of age (HR 0.99, 95\% CI: $0.68-1.48, p=0.979$ ), gender (HR 0.99, 95\% CI: $0.59-1.65, p=0.976$ ), BCLC stage (HR 1.36, 95\% CI: $0.88-$ $2.08, p=0.161$ ), extrahepatic spread (HR 1.06, 95\% CI: $0.72-1.55, p=0.77$ ), and underlying liver disease (viral hepatitis vs. other nonviral hepatitis; HR 0.8, 95\% CI: $0.51-1.24, p=0.313$ ) (Table 3). Patients with macrovascular invasion had a significantly shorter $\mathrm{mOS}$, whereas there was only a trend for a shorter survival of patients with invasion of the main portal vein (HR 1.52; 95\% CI: $0.97-2.38, p=0.063$ ) (Table 3).

Of 160 patients with radiologic staging, $48(30 \%)$ patients were classified as responders and $112(70 \%)$ were classified as nonresponders. Of the responders, 1 patient (2\%) had a CR and 47 (98\%) had a PR, while in nonresponders, 69 (61.6\%) patients experienced disease stabilization and 43 (38.4\%) PD. mOS of responders was significantly longer compared to the nonresponders $(23.9$ months vs. 10.2 months, HR 0.4, 95\% CI: $0.23-0.68, p=$ $<0.001$ ) (Fig. 3; Tables 3, 4).

In addition to radiological evaluation, AFP levels of 192 patients were monitored at baseline and during the treatment. A 20\% reduction in AFP was considered as response, resulting in an overall AFP response in $27.8 \%$ of the patients. In contrast to patients with a radiological response, there was only a trend for a better survival of AFP responders compared to nonresponders (14.5 months vs. 12.7 months, HR 0.83 , 95\% CI: $0.54-1.28$, $p=$ 0.391) (online suppl. Fig. 1a; Table 3). Additionally, AFP response correlated only weakly with radiologic response $(r=0.273, p=0.003)$ (online suppl. Fig. 1b).

\section{Impact of Local Therapy before and Systemic Therapy} after Treatment with Lenvatinib

A total of 148 patients (72.2\%) received local treatment before start of systemic therapy with lenvatinib (Table 1). The most frequent local therapy was TACE, and $87 \mathrm{pa}-$ tients (42.2\%) had received at least 1 and up to 10 TACE treatments prior to lenvatinib (Table 1). The number of TACE treatments before start of lenvatinib had no effect on MPFS and mOS under systemic therapy (mPFS: 6.1 months $<3$ TACE treatments vs. 6.9 months $\geq 3$ TACE 
Table 3. Univariate analysis ( $p$ log-rank) of overall survival

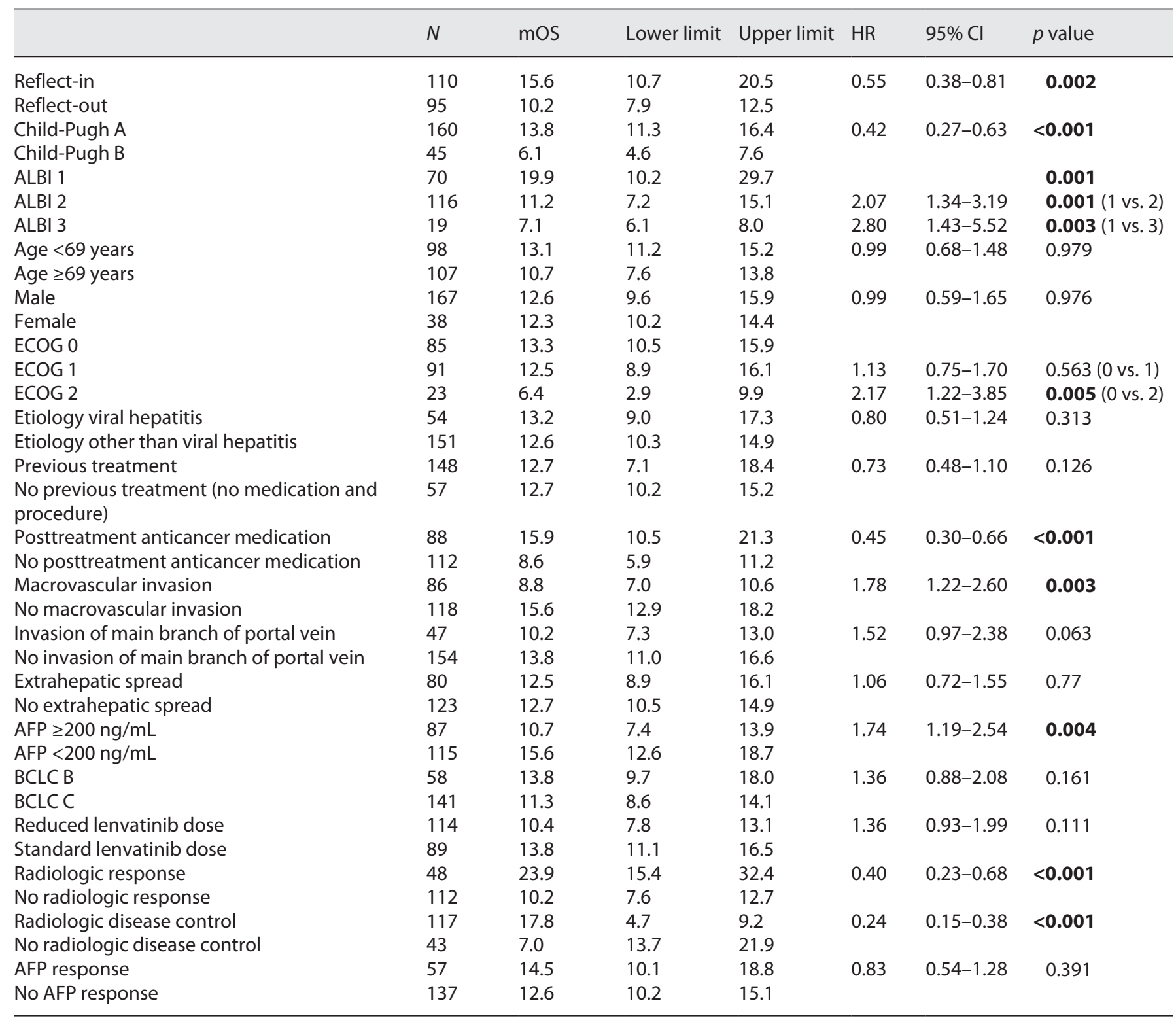

Univariate analysis ( $p$ log-rank) of overall survival in months. $N$, number of patients with available data; median overall survival with lower and upper $95 \% \mathrm{Cl}$ level and $p$ value.

treatments, $p=0.96$; mOS: 11.4 months $<3$ TACE treatments vs. 13.2 months $\geq 3$ TACE treatment; $p=0.42$ ) (online suppl. Fig. 2a, b).

Of all 205 patients, 88 patients (42.93\%) received subsequent second-line systemic anticancer medication, with sorafenib being the most commonly administered second-line treatment $(n=56,63.3 \%)$ followed by cabozantinib ( $n=11,12.5 \%)$, ramucirumab $(n=9,10.2 \%)$, and regorafenib $(n=2,2.3 \%)$. Immunotherapy was given

Real-World Data for Lenvatinib in

Hepatocellular Carcinoma in 11 cases (12.5\%) (pembrolizumab $n=4$ [4.5\%], nivolumab $n=2$ [2.3\%], atezolizumab/bevacizumab $n=$ 3 [3.4\%], and nivolumab/ipilimumab $n=2$ [2.3\%]). Of note, the majority of patients included in ELEVATOR were treated prior to EMA approval of atezolizumab and bevacizumab as first-line systemic treatment for advanced HCC. Subsequent surgical or local-ablative procedures were performed in 9 patients (transplantation [ $n$ $=1]$, SIRT $[n=3]$, radiation $[n=3]$, and ablation $[n=2]$ ) 


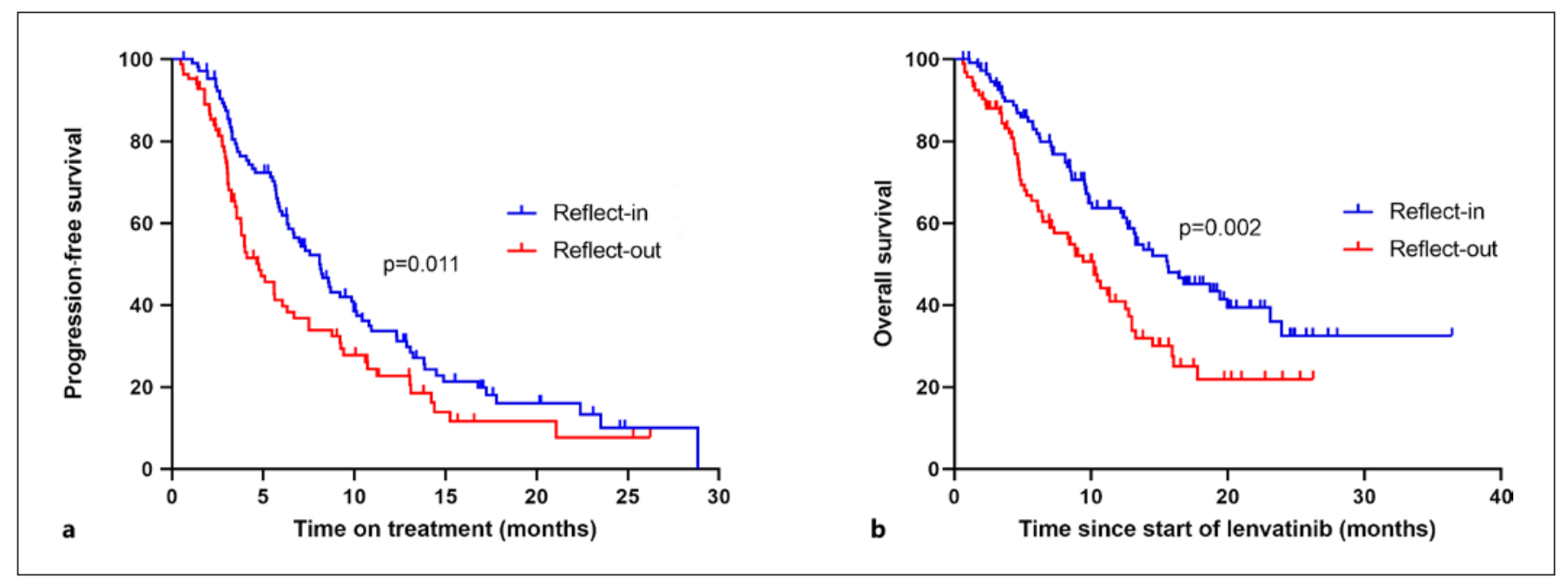

Fig. 1. Kaplan-Meier analysis of progression-free survival (a) and overall survival (b) of REFLECT-in versus REFLECT-out patients.

Table 4. Treatment characteristics according to ALBI score at baseline

\begin{tabular}{|c|c|c|c|c|}
\hline & Overall $(n=205)$ & ALBI $1(n=70)$ & ALBI $2(n=116)$ & ALBI $3(n=19)$ \\
\hline \multicolumn{5}{|l|}{ Best response under lenvatinib treatment } \\
\hline Complete response & $1(0.4)$ & 0 & $1(0.9)$ & 0 \\
\hline Partial response & $47(22.9)$ & $22(31.4)$ & $21(18.2)$ & $4(21.1)$ \\
\hline Stable disease & $69(33.7)$ & $27(38.6)$ & $36(31.0)$ & $6(31.6)$ \\
\hline Progressive disease & $43(20.9)$ & $14(20.0)$ & $23(19.8)$ & $6(31.6)$ \\
\hline End of treatment before first imaging & $38(18.6)$ & $3(4.3)$ & $33(28.4)$ & $2(10.5)$ \\
\hline No data available & $7(3.5)$ & $4(5.7)$ & $2(1.7)$ & $1(5.2)$ \\
\hline Median overall survival, months & 12.8 & 19.9 & 11.2 & 7.1 \\
\hline Median progression-free survival, months & 6.4 & 8.6 & 5.9 & 4.9 \\
\hline Disease control & $117(57.1)$ & $49(70)$ & $58(50.0)$ & $10(52.5)$ \\
\hline Overall response & $48(23.4)$ & $22(31.4)$ & $22(19.0)$ & $4(21.1)$ \\
\hline AFP reduction $>20 \%$ under treatment & $57(27.8)$ & $22(31.4)$ & $31(26.7)$ & $4(21.1)$ \\
\hline \multicolumn{5}{|l|}{ Reason for end of treatment } \\
\hline Progression & $93(45.5)$ & $39(55.8)$ & $43(37.2)$ & $11(57.9)$ \\
\hline Adverse events & $75(36.5)$ & $16(22.8)$ & $52(44.8)$ & $7(36.8)$ \\
\hline Liver function deterioration & $30(14.6)$ & $5(7.1)$ & $21(18.1)$ & $4(21.0)$ \\
\hline Other adverse events & $45(21.9)$ & $11(15.7)$ & $31(26.7)$ & $3(15.8)$ \\
\hline Patient choice & $3(1.4)$ & $1(1.4)$ & $2(1.7)$ & 0 \\
\hline Others & $4(2.0)$ & $1(1.4)$ & $3(2.6)$ & 0 \\
\hline No data available & $6(2.9)$ & $3(4.2)$ & $3(2.6)$ & 0 \\
\hline Treatment ongoing & $24(11.7)$ & $11(15.7)$ & $12(10.3)$ & $1(5.2)$ \\
\hline
\end{tabular}

(online suppl. Table 2). The mOS for patients receiving or not receiving subsequent anticancer medication was 15.9 months versus 8.6 months (HR 0.45, 95\% CI: 0.30 0.66; $p=<0.001$ ) (Table 3; Fig. 4).

\section{Safety and Liver Function}

AEs were documented in $78.1 \%(n=160)$ of patients treated with lenvatinib. No AEs were reported or documented in $21.9 \%(n=45)$. Severe AEs (grade 3 or higher) were reported in $47.3 \%$ of cases. Dose reductions due to poor tolerability were required in 73 cases $(35.6 \%)$ and 


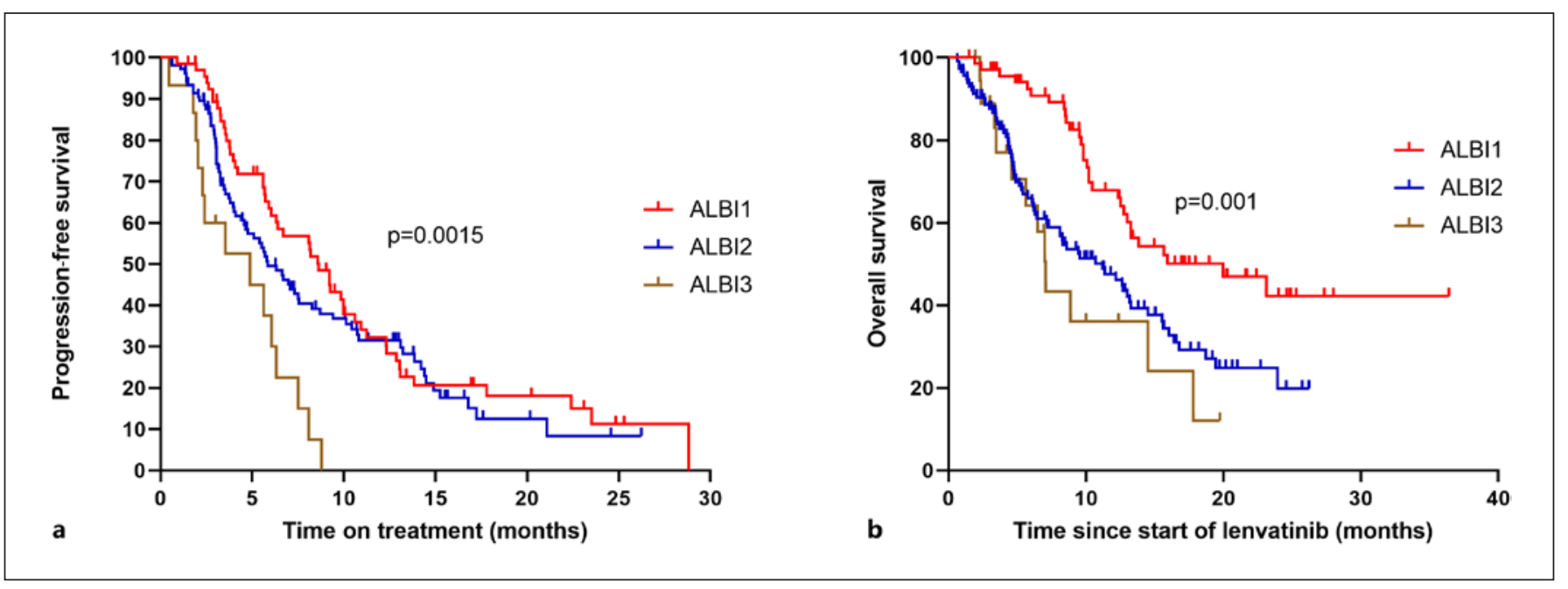

Fig. 2. Kaplan-Meier analysis of progression-free survival (a) and overall survival (b) according to liver function at baseline measured by the ALBI score.

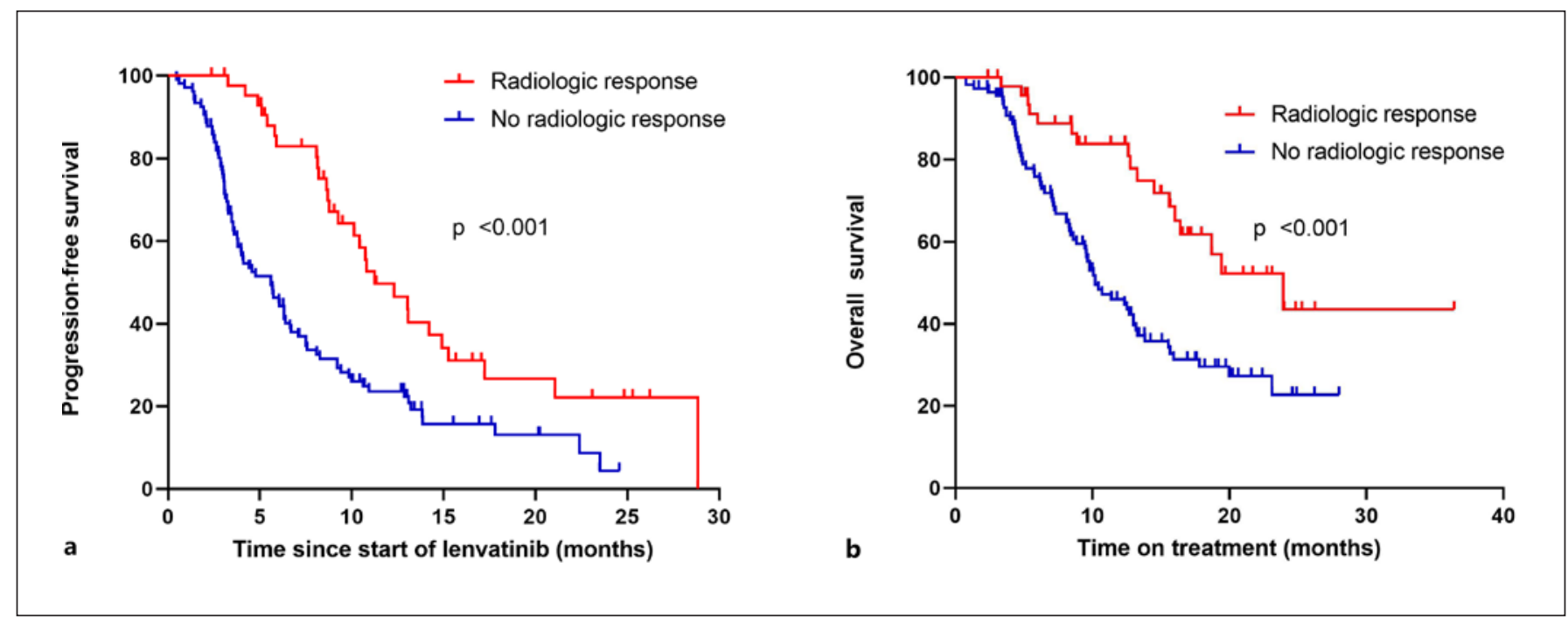

Fig. 3. Kaplan-Meier analysis of progression-free survival (a) and overall survival (b) according to radiologic response under lenvatinib treatment.

treatment interruptions in 63 cases $(30.7 \%)$. The most common documented AE was fatigue $(n=72,35.1 \%)$, loss of appetite $(n=42,20.5 \%)$, diarrhea $(n=38,18.5 \%)$, and weight loss $(n=32,15.6 \%)$. Hypertension was reported in 18 cases $(8.8 \%)$. With respect to liver-related side effects, 34 patients (19.6\%) developed ascites during treatment. Additionally, de novo hepatic encephalopathy was documented in 18 patients $(8.8 \%)$ and classified as grade 3 or 4 in 8

Real-World Data for Lenvatinib in Hepatocellular Carcinoma patients (3.9\%). In 7 out of these 18 patients, ascites was evident at the start of therapy as a surrogate marker of portal hypertension. An impaired liver function corresponding to an ALBI score of 2 or worse was evident in $80 \%$ of these cases (ALBI grade 2: 70\%, ALBI grade 3: 10\%). Frequency of total and severe AEs was comparable in patients with standard and reduced lenvatinib with a trend toward less events under standard dosing (online suppl. Table 3 ). 
Table 5. Uni- and multivariate analysis of overall survival

\begin{tabular}{|c|c|c|c|c|c|c|c|}
\hline & \multirow[t]{2}{*}{$N$} & \multicolumn{3}{|c|}{ Univariate analysis } & \multicolumn{3}{|c|}{ Multivariate analysis } \\
\hline & & $\mathrm{HR}$ & $95 \% \mathrm{Cl}$ & Sig & $H R$ & $95 \% \mathrm{Cl}$ & Sig \\
\hline ALBI1 versus ALBI2 & 186 & 2.07 & $1.34-3.19$ & $<0.001$ & 1.69 & $1.07-2.66$ & 0.023 \\
\hline ALBI1 versus ALBI3 & 89 & 2.80 & $1.43-5.52$ & 0.003 & 1.68 & $0.79-3.61$ & 0.18 \\
\hline ECOG 0 versus ECOG 1 & 176 & 1.13 & $0.75-1.70$ & 0.563 & 1.23 & $0.80-1.89$ & 0.345 \\
\hline ECOG 0 versus ECOG 2 & 108 & 2.17 & $1.22-3.85$ & 0.005 & 2.25 & $1.19-4.23$ & 0.012 \\
\hline Macrovascular invasion & 204 & 1.78 & $1.22-2.60$ & 0.003 & 1.55 & $1.02-2.37$ & 0.041 \\
\hline AFP $\geq 200 \mathrm{ng} / \mathrm{mL}$ & 203 & 1.74 & $1.19-2.54$ & 0.004 & 1.56 & $1.03-2.34$ & 0.034 \\
\hline
\end{tabular}

Uni- and multivariate analysis of overall survival with hazard ratio, corresponding $95 \%$ confidence interval and $p$ value.

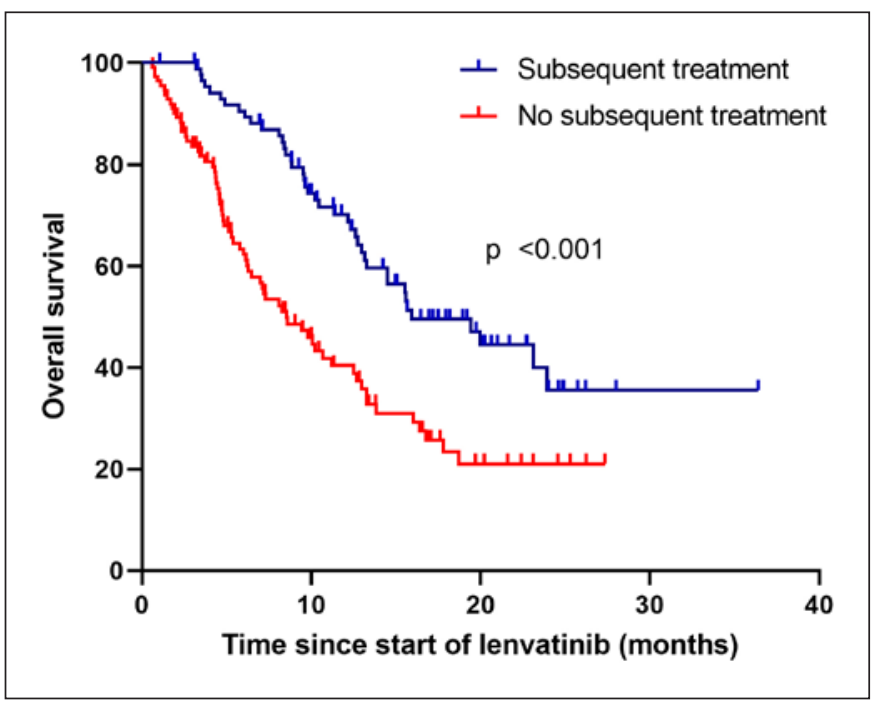

Fig. 4. Overall survival according to subsequent treatment.

To evaluate liver function during treatment with lenvatinib, the ALBI score was calculated at start and end of treatment. In 160 patients with available data, liver function deteriorated in 87 patients (defined as an at least 0.2 -point increase in the score), while in 73 patients, liver function improved ( $n=27$, cutoff 0.2 points) or remained stable $(n=46)$ (online suppl. Fig. $3 b)$. Overall, there was a trend toward a decline of liver function (ALBI grade 1: 54 patients before start of lenvatinib vs. 34 patients at end of therapy, ALBI grade 2: 88 patients before start of lenvatinib vs. 89 patients at end of therapy, ALBI grade 3: 18 patients before start of lenvatinib vs. 37 patients at end of therapy) (online suppl. Fig. 3c). The same trend was observed for the CP score from start to end of treatment (CP A 123 vs. 75 patients, CP B 37 vs. 73 patients, and CP C 0 vs. 12 patients) (online suppl. Fig. 3a, 3d). Overall, the CP score increased by a median of 1 point (mean 1.006 points), while the ALBI score worsened by a median of 0.25 points (mean 0.23 ). However, patients with ALBI grade 2 or 3 at baseline more often discontinued therapy due to deterioration of liver function (ALBI grade 1: 7.1\%; ALBI grade 2: 18.1\%; ALBI grade 3: $21.0 \%$ ) (Table 4). Similarly, the proportion of patients in whom therapy had to be discontinued due to impaired liver function was significantly higher in the REFLECT-out subgroup (9.1\% REFLECT-in vs. 20.1\% REFLECT-out) (Table 2). To determine whether the decline of liver function could rather be attributed to drug-related toxicity or an increase of tumor burden, the 30 patients with therapy discontinuation due to deterioration of liver function were analyzed in further detail. Only two of these patients had a high tumor burden at therapy initiation ( $>50 \%$ intrahepatic tumor invasion), but they remained stable under lenvatinib therapy. In most patients (17/30), liver function deterioration occurred comparatively early (within the first 60 days of treatment), and in patients in whom therapy was terminated due to functional deterioration at a later time point, only a single patient showed PD. Consequent$l y$, in the majority of patients, liver dysfunction was more likely caused by lenvatinib and not by a high tumor burden or tumor progression.

\section{Prognostic Markers for Overall Survival}

Finally, we aimed to identify prognostic factors for patients undergoing first-line treatment with lenvatinib in the ELEVATOR cohort. We observed a significant correlation of OS with the classification according to REFLECT-in and -out (HR 0.55, 95\% CI: 0.38-0.81, $p=$ 0.002), liver function according to ALBI score (ALBI grade 1 vs. ALBI grade 2 HR 2.07, 95\% CI: 1.34-3.19, $p=$ 0.001 ; ALBI grade 1 vs. ALBI grade 3 HR 2.80, 95\% CI: $1.43-5.52, p=0.003$ ) and Child-Pugh score (HR 0.42, 
Fig. 5. Cox multivariate analysis of overall survival.

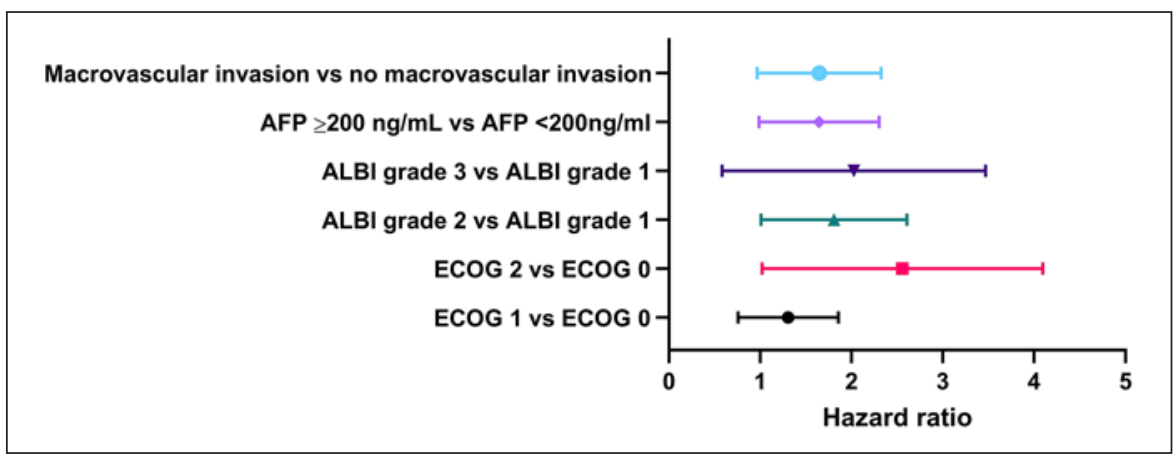

95\% CI: $0.27-0.63, p=<0.001)$, ECOG performance status $\geq 2$ (ECOG 0 vs. ECOG 2 HR 2.17, 95\% CI: 1.22-3.85, $p=0.005$, ECOG 0 vs. ECOG 1 HR 1.13, 95\% CI: 0.75-1.7, $p=0.563$ ), presence of macrovascular invasion (absence vs. presence of macrovascular invasion, HR 1.78, 95\% CI: $1.22-2.60, p=0.003)$, and AFP levels at start of therapy $(\mathrm{AFP}<200 \mathrm{ng} / \mathrm{mL}$ vs. AFP $\geq 200 \mathrm{ng} / \mathrm{mL}, \mathrm{HR} 1.74,95 \% \mathrm{CI}$ : $1.19-2.54, p=0.004$ ) (Table 3). In the multivariate Cox regression analysis, the presence of macrovascular invasion (HR 1.55, 95\% CI: 1.02-2.37, $p=0.041$ ), ECOG performance status 2 (HR 2.25, 95\% CI: 1.19-4.23, $p=0.012$ ), $\mathrm{AFP} \geq 200 \mathrm{ng} / \mathrm{mL}$ (HR 1.56, 95\% CI: $1.03-2.34, p=0.034$ ), and liver function according to ALBI (HR 1.69; 95\% CI: $1.07-2.66, p=0.023$ ) were identified as negative independent prognostic biomarkers in our cohort (Table 5; Fig. 5).

\section{Discussion}

The ELEVATOR study evaluated the safety and efficacy of lenvatinib as first-line systemic treatment in a real-world multicenter cohort comprising 205 patients with advanced HCC. To the best of our knowledge, this is the largest real-world cohort in the first-line setting that includes mainly Caucasian HCC patients [15-18].

Our cohort differed in several baseline criteria characteristics from the pivotal REFLECT trial population, and specifically included more patients with an impaired liver function and reduced ECOG performance status. While portal vein invasion served as an exclusion criterion in the REFLECT trial, these patients made up $22.9 \%$ of our realworld cohort. Moreover, in the REFLECT study, the majority (67\%) of the patients was recruited in Asia, and 73\% of cases were related to viral hepatitis. In contrast, in our predominantly Caucasian cohort, the spectrum of underlying liver disease was more equally distributed between viral hepatitis (28.3\%), alcoholic hepatitis (25.3\%) and nonalcoholic steatohepatitis (20.5\%). Other baseline patient characteristics such as patient age, gender, and body weight in our cohort were similar to the REFLECT trial population. Further, the proportion of advanced stage HCC patients, according to BCLC classification, previous local therapies, and the proportion of patients with extrahepatic spread and an AFP $\geq 200 \mathrm{ng} / \mathrm{mL}$ were comparable between the two cohorts.

Notably, OS in the ELEVATOR cohort was only slightly shorter than in the REFLECT cohort (REFLECT 13.6 months vs. ELEVATOR 12.8 months), and, similarly, the mPFS of 6.4 months ranged slightly behind the PFS data of the REFLECT trial lenvatinib cohort (7.4 months). Considering that $46.3 \%$ of our population would have been excluded from the REFLECT trial, we specifically assessed the survival of patients who would have fulfilled the inclusion criteria. As expected, $\mathrm{mPFS}$ and $\mathrm{mOS}$ in the REFLECT-in subgroup were significantly longer compared to patients that did not meet the inclusion criteria of the prospective clinical trial. In line with this finding, multivariate Cox regression identified liver function, ECOG-PS, and macrovascular invasion as the strongest prognostic marker in our cohort. With respect to etiology, we did not observe a significant difference in efficacy in patients with viral hepatitis compared to any other underlying disease.

The post hoc analysis of the REFLECT trial provided compelling evidence that radiologic response is a surrogate marker for an improved OS. We also observed a strong correlation between radiological response and survival in our real-life cohort, and similar to the REFELCT trial, mOS was twice as long for responders compared to nonresponders [19]. Thus, our analysis further supports the concept that radiologic response serves as an early indicator of a longer mOS [20,21]. Notably, in our cohort, there was only a weak correlation between 
radiologic and AFP response, but considering the retrospective nature of our study, we cannot exclude that this discrepancy might, in part, be attributed to the lack of a coordinated assessment of AFP levels and imaging.

We believe that our study especially provides added value in terms of the efficacy and outcome of lenvatinib treatment in patients with a more impaired liver function, which were excluded from the REFLECT study. A recent post hoc analysis of the phase-III data revealed that, in both the lenvatinib and the sorafenib arm, mOS was longer in patients with a baseline ALBI grade of 1, compared to those with ALBI grade 2 [22]. Additionally, there was a slight trend in the efficacy data, favoring lenvatinib over sorafenib in patients with an ALBI score of 1 . Similarly, in our cohort, efficacy of lenvatinib as assessed by OS, PFS, and ORR was superior in ALBI grade 1 patients compared to patients with a more compromised liver function: mOS of patients with ALBI grade 1 liver function was 19.9 months, which was in a similar range as in the REFLECT population (17.4 months), and also the mOS for the ALBI grade 2 subgroup was comparable between both cohorts. Although the ELEVATOR cohort showed a slightly lower ORR for patients with ALBI 1 liver function, there was a clear difference between ALBI grade 1 and ALBI grade 2 patients in both studies. These data are in line with the strong prognostic impact of liver function in HCC, which has been well documented in retrospective studies, as well as in recent post hoc analyses of the phase-III trials for sorafenib, cabozantinib, ramucirumab, or regorafenib conducted in the first- and second-line setting in advanced HCC $[5,6,15,23-26]$. Overall, our data support the use of the ALBI grade, which is calculated exclusively on the basis of objectively determined laboratory parameters, as a preferred tool to evaluate liver function and more precisely identify patients with only moderate impairment of liver function especially within the Child-Pugh A cohort [27].

The rate of documented AEs in our analysis was 78.1\%, which is significantly lower compared to the REFLECT cohort with $98.7 \%$ [28]. However, due to the retrospective data collection, underreporting in the real-life setting has to be considered as a potential confounder of these results. In our cohort, the proportion of AEs and severe AEs in patients receiving the standard dosing regimen was similar to what was reported in patients who were started on lenvatinib at a reduced dose. Therefore, we currently do not have evidence that the lower rate of AEs can be attributed to the reduced dose. Serious treatment-associated side effects, defined as grade 3 or higher, occurred in $47.3 \%$, which is comparable to the REFLECT trial (56.7\%)
[22]. Of note, in about one-third of cases in our cohort, AEs, including deterioration of liver function, accounted for therapy discontinuation. In the REFLECT-out subgroup, this proportion was even higher with $45 \%$. Similar to the REFLECT data, severe AEs grade $>3$ occurred more frequently in patients with more compromised liver function [22]. Overall, our data are in support of the post hoc analysis of the REFLECT trial and indicate that patients with a preserved liver function and an ECOG-PS $\leq 1$ tolerate lenvatinib treatment better and are at lower risk of premature treatment discontinuation. However, in clinical practice, a considerable proportion of patients with advanced HCC does not fulfill the inclusion criteria of a clinical trial, and in the real-world setting, it is not uncommon that lenvatinib treatment is initiated at a lower than recommended dose. Similar to the observations published for sorafenib, our data suggest that a reduced lenvatinib dosage may be a safe and a reasonable strategy for some patients with HCC [29].

Deterioration of liver function is a clinically relevant problem for HCC patients during the course of local and systemic treatments. In our cohort, several patients stopped treatment due to hepatic decompensation with either encephalopathy or ascites. Overall, liver function deteriorated in $54.4 \%(87 / 160)$ of evaluable patients. It should be noted that a decline of liver function can be multifactorial and may be related to the underlying liver disease, tumor progression, and/or side effects of therapy. Of note, baseline liver function did not correlate with the frequency or the severity of further deterioration (according to the numeric increase of the ALBI score) during the course of therapy. However, within the CP A population, $21.5 \%$ of the ALBI grade 1 patients and $39 \%$ of the ALBI grade 2 patients had transitioned to $\mathrm{CP} B$ at the time of treatment discontinuation. Similarly, patients with an ALBI grade 2/CP A at baseline were at higher risk to deteriorate to $\mathrm{CP} \mathrm{B}$ cirrhosis in the REFLECT study. Although CP B liver function may improve in a small fraction of patients after termination of therapy, entering $\mathrm{CP}$ $\mathrm{B}$ stage decreases the likelihood to receive subsequent anticancer treatment.

Recent analysis from the global phase-III studies in the first-line setting indicated that up to $50 \%$ of patients in clinical trials are amenable to second-line therapy in advanced HCC $[28,30]$. Accordingly, in our real-world cohort, $43 \%$ of patients received subsequent systemic therapy after lenvatinib, as recommended by international guidelines [31]. In line with the data from the REFLECT study, sorafenib was the drug that was most frequently used after lenvatinib in our analysis, and mOS was sig- 
nificantly longer for patients that were able to receive sequential therapy [32]. In addition, small real-world cohorts suggest that the efficacy of lenvatinib is comparable in first and later lines of systemic therapy [33]. Currently, there is no standard second-line therapy established for patients that did not receive sorafenib in the first-line setting, and especially after the recent EMA approval of atezolizumab and bevacizumab, additional studies are required to delineate the optimal systemic treatment sequences in HCC. Real-world analyses will likely complement the evidence from phase-III studies in favor of second- and higher-line treatment in HCC [4-6] and will contribute to a better understanding of sequential treatments in HCC.

ELEVATOR was designed as a multicenter study, recruiting patients from 14 sites in Germany and Austria. However, limitations include its retrospective nature and the lack of independent review of treatment responses by RECIST 1.1 and mRECIST. Additionally, radiological assessment of treatment response according to the defined RECIST 1.1 criteria was not documented for all patients and was not performed at predefined time points. Additionally, we were not able to provide the exact dose intensity for lenvatinib. Further, the subgroup analyses contained a limited number of patients, and additional studies are needed to validate our findings.

In aggregate, our data confirm the efficacy of lenvatinib as first-line treatment and did not reveal new or unexpected side effects in a large retrospective Caucasian real-world cohort. The analyses indicate that patients who do not meet the inclusion criteria of the REFLECT trial may benefit from lenvatinib therapy but could be at higher risk for side effects and deterioration to ChildPugh B cirrhosis. Therefore, close clinical and laboratory monitoring is advised. Our data support the use of lenvatinib as first line systemic therapy for HCC patients that cannot be treated with IO-based combinations.

\section{Statement of Ethics}

This study protocol was reviewed and approved by the Ethics Committee of the Medical School Hannover, Hannover, Germany, Approval No. 940-2011. Written informed consent was obtained from all participants, and the study was conducted ethically in accordance with the World Medical Association Declaration of Helsinki.

\section{Conflict of Interest Statement}

Honoraria for speaker, consultancy, and advisory role to A.V.: Roche, Bayer, Sanofi, BMS, Lilly, Novartis, EISAI, AstraZeneca, Merck, Ipsen, PierreFabre, and MSD. Honoraria for speaker, consultancy, and advisory role to A.S.: Roche, BMS, and Servier. Honoraria for speaker, consultancy, and advisory role to M.A.G.C.: Roche, Bayer, EISAI, MSD, and Servier. Honoraria for speaker, consultancy, and advisory role to C.M.L.: Roche, Novartis, EISAI, MSD, CSL Behring, AbbVie, and Gilead. Honoraria for speaker to C.L.: EISAI. Honoraria for speaker role to P.J.: Ipsen. Honoraria for speaker and advisory role to U.E.: AstraZeneca, Bayer, EISAI, Ipsen, Lilly, Novartis, Roche, and SIRTEX. Honoraria for speaker and advisory role to A.W.: Bayer, BMS, Eisai and Sanofi, Leo Pharma, Eisai, Ipsen, and Roche, travel support from Merck and Servier, A.W. is an Editorial Board Member of Liver Cancer. A.J. received speaker fees from AbbVie, MSD, Eisai, and Roche; travel grant from Servier, BMS, Amgen, and Lilly, sponsoring from Lilly, Cellgene, and Amgen, and personal fees from Servier, Amgen, Eisai, and Bayer. Honoraria for speaker and advisory board to A.K.: Roche Pharma AG; Eisai GmbH; Abbvie Deutschland, JanssenCilag; Boston Scientific Corp.; Micro-Tech Europe. Honoraria for speaker and advisory role to R.G.: Ad-Boards Bayer, Eisai/MSD, Ipsen, and Roche. Travel support and honoraria for speaker to F.F.: Abbvie, Ipsen, and Fresenius. Honoraria for speaker, consultancy, and advisory role to O.W.: Amgen, Bayer, BMS, Celgene, Eisai, Incyte, Ipsen, Merck Serono, MSD, Novatis, Roche, Servier, Shire, AstraZeneca, Abbvie, Gilead, and Medac. Honoraria for speaker, consultancy, and advisory role to M.V.: Roche, BMS, EISAI, Bayer, Lilly, AstraZeneca, Merck Serono, Sirtex, MSD, Ipsen, Nordic Pharma, and Amgen. Honoraria for speaker, consultancy, and advisory role to J.B.: Roche, BMS, EISAI, MSD, Novartis, and Ipsen. Honoraria for speaker, consultancy, and advisory role to A.D.: Roche, Bayer, Lilly, Novartis, EISAI, AstraZeneca, Merck, Ipsen, PierreFabre, Service, and Merck.

\section{Funding Sources}

Funding was provided by EISAI (documentation fee for the patients included in the study).

\section{Author Contributions}

S.W., A.S., and A.V. designed and performed research, analyzed and interpreted data, and wrote the manuscript. C.L., F.F., A.J., K.S., M.G.-C., A.K., P.J., T.W.F., M.P., A.M., C.F., R.G., H.W., U.E., M.M., C.P.S., A.W., J.S., O.W., J.B., M.V., A.D., A.S., R.G., and C.M.L contributed to acquisition of data and critical revision of the manuscript for important intellectual content.

\section{Data Availability Statement}

All data generated or analyzed during this study are included in this article and its online supplementary material files. Further enquiries can be directed to the corresponding author.
Real-World Data for Lenvatinib in

Hepatocellular Carcinoma
Liver Cancer 2022;11:219-232

DOI: $10.1159 / 000521746$ 


\section{References}

1 Sung H, Ferlay J, Siegel RL, Laversanne M, Soerjomataram I, Jemal A, Bray F. Global Cancer Statistics 2020: GLOBOCAN Estimates of Incidence and Mortality Worldwide for 36 Cancers in 185 Countries. CA Cancer J Clin. 2021 May;71(3):209-49.

2 Ferlay J, Colombet M, Soerjomataram I, Mathers C, Parkin DM, Piñeros M, et al. Estimating the global cancer incidence and mortality in 2018: GLOBOCAN sources and methods. Int J Cancer. 2019 Apr 15;144(8):1941-53.

3 Ferlay J, Soerjomataram I, Dikshit R, Eser S, Mathers C, Rebelo M, et al. Cancer incidence and mortality worldwide: sources, methods and major patterns in GLOBOCAN 2012. Int J Cancer. 2015 Mar 1;136(5):359.

4 Bruix J, Qin S, Merle P, Granito A, Huang YH, Bodoky G, et al. Regorafenib for patients with hepatocellular carcinoma who progressed on sorafenib treatment (RESORCE): a randomised, double-blind, placebo-controlled, phase 3 trial. Lancet. 2017 Jan 7;389(10064): 56-66.

5 Kelley RK, Ryoo BY, Merle P, Park JW, Bolondi L, Chan SL, et al. Second-line cabozantinib after sorafenib treatment for advanced hepatocellular carcinoma: a subgroup analysis of the phase 3 CELESTIAL trial. ESMO Open. 2020 Aug;5(4):e000714.

6 Zhu AX, Kang YK, Yen CJ, Finn RS, Galle PR, Llovet JM, et al. Ramucirumab after sorafenib in patients with advanced hepatocellular carcinoma and increased $\alpha$-fetoprotein concentrations (REACH-2): a randomised, doubleblind, placebo-controlled, phase 3 trial. Lancet Oncol. 2019 Feb;20(2):282-96.

7 Llovet JM, Ricci S, Mazzaferro V, Hilgard P, Gane E, Blanc JF, et al. Sorafenib in advanced hepatocellular carcinoma. N Engl J Med. 2008 Jul 24;359(4):378-90.

8 Tohyama O, Matsui J, Kodama K, Hata-Sugi N, Kimura T, Okamoto K, et al. Antitumor activity of lenvatinib (e7080): an angiogenesis inhibitor that targets multiple receptor tyrosine kinases in preclinical human thyroid cancer models. J Thyroid Res. 2014;2014:638747.

9 Finn RS, Qin S, Ikeda M, Galle PR, Ducreux M, Kim TY, et al. Atezolizumab plus bevacizumab in unresectable hepatocellular carcinoma. N Engl J Med. 2020 May 14;382(20): 1894-905.

10 Sonbol MB, Riaz IB, Naqvi SAA, Almquist DR, Mina S, Almasri J, et al. Systemic therapy and sequencing options in advanced hepatocellular carcinoma: a systematic review and network meta-analysis. JAMA Oncol. 2020 Dec 1;6(12):e204930.

11 Vogel A, Rimassa L, Sun HC, Abou-Alfa GK, El-Khoueiry A, Pinato DJ, et al. Comparative efficacy of atezolizumab plus bevacizumab and other treatment options for patients with unresectable hepatocellular carcinoma: a network meta-analysis. Liver Cancer. 2021 Jun; 10(3):240-8.
12 Vogel A, Cervantes A, Chau I, Daniele B, Llovet JM, Meyer T, et al. Hepatocellular carcinoma: ESMO clinical practice guidelines for diagnosis, treatment and follow-up. Ann Oncol. 2019 May 1;30(5):871-3.

13 Ikeda M, Sung MW, Kudo M, Kobayashi M, Baron AD, Finn RS, et al. A phase $1 \mathrm{~b}$ trial of lenvatinib (LEN) plus pembrolizumab (PEM) in patients (pts) with unresectable hepatocellular carcinoma (uHCC). J Clin Oncol. 2018; 36(15):4076.

14 Oken MM, Creech RH, Tormey DC, Horton J, Davis TE, McFadden ET, et al. Toxicity and response criteria of the Eastern cooperative oncology group. Am J Clin Oncol. 1982 Dec; 5(6):649-55.

15 Hiraoka A, Kumada T, Atsukawa M, Hirooka M, Tsuji K, Ishikawa T, et al. Prognostic factor of lenvatinib for unresectable hepatocellular carcinoma in real-world conditions-multicenter analysis. Cancer Med. 2019 Jul;8(8): 3719-28.

16 Hiraoka A, Kumada T, Tada T, Fukunishi S, Atsukawa M, Hirooka M, et al. Nutritional index as prognostic indicator in patients receiving lenvatinib treatment for unresectable hepatocellular carcinoma. Oncology. 2020; 98(5):295-302.

17 Cheon J, Chon HJ, Bang Y, Park NH, Shin JW, Kim KM, et al. Real-world efficacy and safety of lenvatinib in Korean patients with advanced hepatocellular carcinoma: a multicenter retrospective analysis. Liver Cancer. 2020 Sep;9(5):613-24.

18 Goh MJ, Oh JH, Park Y, Kim J, Kang W, Sinn $\mathrm{DH}$, et al. Efficacy and safety of lenvatinib therapy for unresectable hepatocellular carcinoma in a real-world practice in Korea. Liver Cancer. 2021 Feb;10(1):52-62.

19 Alsina A, Kudo M, Vogel A, Cheng AL, Tak WY, Ryoo BY, et al. Effects of subsequent systemic anticancer medication following firstline lenvatinib: a post hoc responder analysis from the phase 3 reflect study in unresectable hepatocellular carcinoma. Liver Cancer. 2020 Jan;9(1):93-104.

20 Kudo M, Ueshima K, Chiba Y, Ogasawara S, Obi S, Izumi N, et al. Objective response by mRECIST is an independent prognostic factor for overall survival in hepatocellular carcinoma treated with sorafenib in the silius trial. Liver Cancer. 2019 Nov;8(6):505-19.

21 Lencioni R, Montal R, Torres F, Park JW, Decaens T, Raoul JL, et al. Objective response by mRECIST as a predictor and potential surrogate end-point of overall survival in advanced HCC. J Hepatol. 2017 Jun;66(6): 1166-72.

22 Vogel A, Qin S, Kudo M, Su Y, Hudgens S, Yamashita T, et al. Lenvatinib versus sorafenib for first-line treatment of unresectable hepatocellular carcinoma: patient-reported outcomes from a randomised, open-label, noninferiority, phase 3 trial. Lancet Gastroenterol Hepatol. 2021 Jun 1.
23 Abdel-Rahman O. Impact of baseline characteristics on outcomes of advanced HCC patients treated with sorafenib: a secondary analysis of a phase III study. J Cancer Res Clin Oncol. 2018 May;144(5):901-8.

24 Bruix J, Cheng AL, Meinhardt G, Nakajima K, De Sanctis Y, Llovet J. Prognostic factors and predictors of sorafenib benefit in patients with hepatocellular carcinoma: analysis of two phase III studies. J Hepatol. 2017 Nov; 67(5):999-1008.

25 Raoul JL, Bruix J, Greten TF, Sherman M, Mazzaferro V, Hilgard P, et al. Relationship between baseline hepatic status and outcome, and effect of sorafenib on liver function: SHARP trial subanalyses. J Hepatol. 2012 May;56(5):1080-8.

26 Cosma LS, Weigand K, Müller-Schilling M, Kandulski A. Lenvatinib as first-line treatment of hepatocellular carcinoma in patients with impaired liver function in advanced liver cirrhosis: real world data and experience of a tertiary hepatobiliary center. J Gastrointestin Liver Dis. 2021 Jun 18;30(2):247-53.

27 Hiraoka A, Kumada T, Atsukawa M, Hirooka M, Tsuji K, Ishikawa T, et al. Prognostic factor of lenvatinib for unresectable hepatocellular carcinoma in real-world conditions-multicenter analysis. Cancer Med. 2019 Jul;8(8): 3719-28.

28 Kudo M, Finn RS, Qin S, Han KH, Ikeda K, Piscaglia F, et al. Lenvatinib versus sorafenib in first-line treatment of patients with unresectable hepatocellular carcinoma: a randomised phase 3 non-inferiority trial. Lancet. 2018 Mar 24;391(10126):1163-73.

29 Alghamdi MA, Amaro CP, Lee-Ying R, Sim $\mathrm{HW}$, Samwi H, Chan KK, et al. Effect of sorafenib starting dose and dose intensity on survival in patients with hepatocellular carcinoma: results from a Canadian multicenter database. Cancer Med. 2020 Jul;9(14):4918-28.

30 Finn RS, Qin S, Ikeda M, Galle PR, Ducreux M, Kim TY, et al. Atezolizumab plus bevacizumab in unresectable hepatocellular carcinoma. N Engl J Med. 2020 May 14;382(20): 1894-905.

31 Vogel A, Cervantes A, Chau I, Daniele B, Llovet JM, Meyer T, et al. Hepatocellular carcinoma: ESMO clinical practice guidelines for diagnosis, treatment and follow-up. Ann Oncol. 2018 Oct 1;29(Suppl 4):iv238-iv255.

32 Alsina A, Kudo M, Vogel A, Cheng AL, Tak WY, Ryoo BY, et al. Effects of subsequent systemic anticancer medication following firstline lenvatinib: a post hoc responder analysis from the phase 3 REFLECT study in unresectable hepatocellular carcinoma. Liver Cancer. 2020 Jan;9(1):93-104.

33 Jefremow A, Wiesmueller M, Rouse RA, Dietrich P, Kremer AE, Waldner MJ, et al. Beyond the border: the use of lenvatinib in advanced hepatocellular carcinoma after different treatment lines: a retrospective analysis. J Physiol Pharmacol. 2020 Oct;71(5):10. 